\title{
Effect of Nitrogen and Potassium Fertilizer Application at Physiological Growth Stages on Yield of Two Wheat Varieties Grown in the North Middle Delta
}

\author{
Faizy, S. E.D. A.; M. M. RizK' ${ }^{1}$ E.A.E.Gazia and M. M.A. Amer ${ }^{2}$
}

\begin{abstract}
Field experiment was carried out at the experimental farm, faculty of Agric., Kafr El Sheikh Univ. during the two successive seasons (2003 and 2004). The objective of the study was to study effect the of $\mathbf{N}$ application at tillering stage on wheat (Triticum aestivum) yield, its components and harvest index (HI), as well as the effect of $\mathrm{K}$. The obtained results could be summarized as follows:

The grain and straw yields of the two studied wheat varieties was highly significantly increased with increasing $\mathrm{N}$ level up to $120 \mathrm{~kg} \mathrm{~N} / \mathrm{fed}$. $\left(\mathrm{fed}=4200 \mathrm{~m}^{2}\right.$ ) The highest mean values were 21.91 and 30.0 ardab grain/fed., $($ ardab $=150 \mathrm{~kg})$ and 5.19 and 6.0 tons straw/fed. for Giza 168 and Sids7, respectively. The number of spikes $/ \mathrm{m}^{2}$, number of grains per spike and 1000-grain weight were highly significantly increased with increasing $\mathrm{N}$ and $\mathrm{K}$ levels. The highest mean value over the two seasons (435 and 300$)$, (83 and 103.5) and $(40.45$ and $62.9 \mathrm{~g})$ for Giza 168 and Sids7 respectively. $\mathrm{N}$ application at tillering stage in the presence of $\mathrm{K}$ have a high significant effect on grain and straw yield, number of spikes/m2, number of grains per spike and 1000-grain weight of the two wheat varieties. The highest mean values were recorded at D6. Hence, application of $K$ lead to increasing $N \mathrm{U}$ E.The maximum 1000-grains weight was 54 g could be obtained at about $36 \mathrm{~kg} N /$ fed. without $K$ addition, while, in the presence of $K$ it was $59 \mathrm{~g}$ and could be obtained at $29 \mathrm{~kg} \mathrm{~N} / \mathrm{fed}$. The highest mean values of $\mathrm{HI}$ were 41.21 and $42.26 \%$ for Giza 168 and Sids7 respectively. $K$ increased the harvest index and were $1.88 \%$ and $2.31 \%$ for Giza 168 and Sids7. It is recommended to apply $120 \mathrm{~kg} \mathrm{~N} / \mathrm{fed}$. as 24, 60, 18 and 18 at planting, tillering, booting and milking stages, respectively as well as applications of $50 \mathrm{~kg} \mathrm{~K} \mathrm{~K}_{2} \mathrm{O} /$ fed. and $22 \mathrm{~kg} \mathrm{P}_{2} \mathrm{O}_{5} / \mathrm{fed}$. before planting. to obtain the maximum values of grain and straw yield for the studied wheat varieties Giza 168 and Sids7 grown under North Middle Delta conditions.
\end{abstract}

\section{INTRODUCTION}

Wheat (Triticum aestivum) is strategic crop because of its indispensable part of food diet. It is widely distributed all over the country (2.987 million feddan in 2008), with a mean production of about 2.745 tons/feddan. The national production of wheat is 8.2 million tons/2008 and the national consumption of wheat is about 12.0 million tons in 2008; there is a great gab between the consumption and production. Reducing the gap between production and consumption of wheat is a national policy in Egypt. Wheat is very sensitive to insufficient $\mathrm{N}$.Application of $\mathrm{N}$ for wheat as one dose was usually practiced in the past. It is found that increasing $\mathrm{N}$ fertilizer rate up to $100 \mathrm{Kg} \mathrm{N} /$ fed increased number of grains/spike, grain, straw and biological yields (Abd-Elwahed 1997). Cox et al (1989) found that addition of $\mathrm{N}$ fertilizer in 2 doses were better. Recently, Faizy 1986a found that addition of $\mathrm{N}$ as three doses gave higher yield. Forestar (1973) found that, the grain and straw yields of wheat increased when the $\mathrm{N}$ fertilizer was splited into two or three doses. Moreover, the addition of four $\mathrm{N}$ doses gave higher yield than $3 \mathrm{~N}$ doses (Rahman et al 2002). Also, Faizy et al. (1986b) found that, the grain yield of wheat was higher when the nitrogen was split into 4 doses than when split into 3 or 2 doses. Nitrogen use Efficiency has been considerably improved by splitting $\mathrm{N}$ dressing in winter wheat (Faizy et al., $1986 \mathrm{~b}$ and Mengel, 2001). Ministry of agricultural recommends applying $\mathrm{N}$ as 3 equal doses.

Potassium is necessary for plant growth, where it plays an integral part. Enzymes are involved in many important plant physiological processes, and over 80 plant enzymes require $\mathrm{K}$ for their activation. $\mathrm{K}$ fertilization has a role in decreasing certain plant diseases and improving quality. Thus, the intensive agriculture reduced the level of available $\mathrm{K}$ in the soil; nowadays insufficient Soil $\mathrm{K}$ is usually corrected by adding $\mathrm{K}$ fertilizer which has a beneficial influence on the development of endosperm cells and hence on the single grain weight of cereals (Schacherer and Beringer 1984). Faizy et al. (1986 b) found that the grain yield of wheat, in the presence of $\mathrm{K}$, was increased significantly at the low and high levels of $\mathrm{N}$. The increase in grain yield by $\mathrm{K}$ fertilizer was 5.91 to $7.29 \%$,. In field experiments in Egypt, the response of 6 wheat cultivars (Giza 162, Giza 163, Giza 164 Gimmeza 1, Sakha 69 and Sids 1) to four levels from K fertilizer $(12,24,36$ and $48 \mathrm{Kg} \mathrm{K}_{2} \mathrm{O} /$ fed.) was studied and their effects on grain yield and some contributing characters. $\mathrm{K}$ rate affects all studied characters of wheat cultivars. The interaction effects of wheat cultivars and $\mathrm{K}$ fertilizer rate were significant on 1000 grain weight, straw and biological yields. Gennady and Hegazy (2001) that 24 $\mathrm{Kg} \mathrm{K}{ }_{2} \mathrm{O} /$ fed as $\mathrm{K}_{2} \mathrm{SO}_{4}$ fertilizer increased the yield of

\footnotetext{
${ }^{1}$ Soil Dept. Fac. of Agric., Kafr EL-Sheikh Univ

${ }^{2}$ Soils, Water and Environment Research Institute, A R C.

Received January8, 2011, Accepted March 4, 2011
} 
wheat by $20.76 \%$.Therefore, the main target of this investigation to study the effect of $\mathrm{N}$ and $\mathrm{K}$ application was found at tillering growth stage on wheat yield and its components and harvest index (HI) of two wheat varieties Giza 168 and Sids7.

\section{MATERIALS AND METHODS}

Field experiment was carried out at the experimental farm, faculty of Agric., Kafr El Sheikh Univ. (6 m altitude, $31^{\circ} 07$ - latitude and $30^{\circ}$ 52- longitude) during two successive seasons (2002/2003 and 2003/2004) with two wheat varieties: Giza 168 and Sids 7, to study the effect of $\mathrm{N}, \mathrm{K}$ timing of $\mathrm{N}$ application at planting, tillering, booting and milk stages on grain and straw yields, number of spikes $/ \mathrm{m}^{2}$, number of grains per spike and 1000 grain weight. Harvest index (HI) was calculated as follows:

The experiments were conducted in split - split - split plot design, with four replicates. The main plots were assigned to timing of $\mathrm{N}$ application. Sub plots were assigned to $\mathrm{N}$ level ( 75 and $120 \mathrm{~kg} / \mathrm{fed}$.), sub- sub plot assigned to $\mathrm{K}$ level (with and without $50 \mathrm{Kg} \mathrm{K}_{2} \mathrm{O} / \mathrm{fed}$.) Sub-sub-sub plots assigned to wheat variety. The plot area was $10.5 \mathrm{~m}^{2}(1 / 400 \mathrm{fed})$. The $\mathrm{N}$ was applied as urea $(46 \% \mathrm{~N})$ in four doses. The first dose was broadcasted with $22 \mathrm{Kg} \mathrm{P}_{2} \mathrm{O}_{5}$ / fed. at sowing. The second dose of $\mathrm{N}$ was applied at the first irrigation (tillering stage) with levels: $0,20,30,40,50,60 \mathrm{Kg}$ N/fed and represented as: $\mathrm{D}_{1}, \mathrm{D}_{2}, \mathrm{D}_{3}, \mathrm{D}_{4}, \mathrm{D}_{5}$ and $\mathrm{D}_{6}$. The rest of $\mathrm{N}$ was splited equally between booting and milking development stages, Table 2 show the Layout of the Experiment.

Soil samples, from the surface layers (0-15 and 1530), were taken from the experimental sites before planting of the two seasons and prepared for physical and chemical analysis. The soil characteristics were presented in Table 1. Also, plant samples were taken randomly at age 45 and 90 day to estimate the wheat Yield and its components. Harvest index, was calculated. Soil samples were air-dried crushed and passed through $2.0 \mathrm{~mm}$ sieve for the chemical analysis according to Jackson (1967). Cation exchange capacity (CEC) as described by Gohar (1954). Available P according to Olsen et al. (1954). and K according to Page (1982). Mechanical analysis was determined according to Piper (1950) Total N content by microKjeldahl method (Jackson, 1967). Total Pwas determined according to Snell and Snell, (1967). Total K content using flame photometer (Jackson 1967). Protein concentration (\%): was calculated from total $\mathrm{N}$ in grain yield multiplied by 6.25 according A. O. A. C. (1980). Data were analyzed statistically according to procedures outlined by Cochran and Cox (1960).

$$
\text { Harvest index }=\frac{\text { Grain yield }}{\text { grain yield }+ \text { straw yield }} \quad \begin{aligned}
& 100 \\
& 0
\end{aligned}
$$

\section{Table 1. Chemical and physical properties of soil surface layer $(0-15,15-30)$ before growing} seasons

\begin{tabular}{|c|c|c|c|c|c|}
\hline \multirow{2}{*}{\multicolumn{2}{|c|}{$\begin{array}{c}\text { Growing seasons } \\
\text { Soil depth } \mathbf{c m} \\
\end{array}$}} & \multicolumn{2}{|c|}{$2002 / 2003$} & \multicolumn{2}{|c|}{ 2003/2004 } \\
\hline & & $0-15$ & $15-30$ & $0-15$ & $15-30$ \\
\hline \multicolumn{2}{|l|}{ Soil pH } & 8.0 & 8.1 & 8.1 & 8.13 \\
\hline \multicolumn{2}{|l|}{$E C d_{S m}^{-1}$} & 2.3 & 3.6 & 2.0 & 2.5 \\
\hline \multicolumn{2}{|l|}{ SAR } & 4 & 7 & 5.2 & 6.8 \\
\hline \multirow{3}{*}{$\begin{array}{c}\text { Available } \\
\text { ppm }\end{array}$} & $\mathbf{N}$ & 32 & 17 & 30 & 18 \\
\hline & $\mathbf{P}$ & 12 & 10 & 11 & 10 \\
\hline & $\mathbf{K}$ & 195 & 150 & 201 & 159 \\
\hline \multicolumn{2}{|l|}{ O.M \% } & 2.36 & 2.18 & 2.41 & 2.20 \\
\hline \multicolumn{2}{|l|}{ Total N\% } & 0.17 & 0.16 & 0.18 & 0.15 \\
\hline \multicolumn{2}{|c|}{ Total carbonate \% } & 3.6 & 3.57 & 3.55 & 3.4 \\
\hline \multicolumn{2}{|c|}{ CEC meq/100g soil } & 45.2 & 43.1 & 47.1 & 44.6 \\
\hline \multicolumn{2}{|c|}{ Saturation percent } & 93 & 90 & 91 & 89 \\
\hline \multirow{3}{*}{ Particle size distribution \% } & Clay & 57.2 & 58.11 & 56.90 & 56.71 \\
\hline & Silt & 32.0 & 31.41 & 30.29 & 30.90 \\
\hline & Sand & 10.8 & 10.48 & 12.81 & 12.39 \\
\hline \multicolumn{2}{|l|}{ Texture grade } & clayey & clayey & clayey & clayey \\
\hline
\end{tabular}


Table 2. Layout of the Experiment

\begin{tabular}{|c|c|c|c|c|c|c|c|}
\hline \multicolumn{8}{|c|}{ TREATMENTS (Kg / fed.) } \\
\hline \multirow{2}{*}{$\mathbf{D}$} & \multirow{2}{*}{ N Level } & \multicolumn{4}{|c|}{ Different doses of $\mathrm{N}$ at physiological growth stages } & \multirow{2}{*}{$\frac{\mathbf{P}}{\mathbf{P}_{2} \mathbf{O}_{5}}$} & \multirow{2}{*}{\begin{tabular}{c|}
$\mathrm{K}$ \\
$\mathrm{K}_{2} \mathrm{O}$
\end{tabular}} \\
\hline & & Planting & Tillering & Booting & Milk & & \\
\hline Cont. & $\mathbf{0}$ & 0 & 0 & 0 & 0 & 0 & 0 \\
\hline D1 & 75 & 15 & 0 & 30 & 30 & 22 & $\begin{array}{c}0 \\
50\end{array}$ \\
\hline D2 & 75 & 15 & 20 & 20 & 20 & 22 & $\begin{array}{c}0 \\
50\end{array}$ \\
\hline D3 & 75 & 15 & 30 & 15 & 15 & 22 & $\begin{array}{c}0 \\
50\end{array}$ \\
\hline D4 & 75 & 15 & 40 & 10 & 10 & 22 & $\begin{array}{c}0 \\
50\end{array}$ \\
\hline D5 & 75 & 15 & 50 & 5 & 5 & 22 & $\begin{array}{c}0 \\
50\end{array}$ \\
\hline D6 & 75 & 15 & 60 & 0 & 0 & 22 & $\begin{array}{c}0 \\
50\end{array}$ \\
\hline D1 & 120 & 24 & 0 & 48 & 48 & 22 & $\begin{array}{c}0 \\
50\end{array}$ \\
\hline D2 & 120 & 24 & 20 & 38 & 38 & 22 & $\begin{array}{c}0 \\
50\end{array}$ \\
\hline D3 & 120 & 24 & 30 & 33 & 33 & 22 & $\begin{array}{c}0 \\
50\end{array}$ \\
\hline D4 & 120 & 24 & 40 & 28 & 28 & 22 & $\begin{array}{c}0 \\
50\end{array}$ \\
\hline D5 & 120 & 24 & 50 & 23 & 23 & 22 & $\begin{array}{c}0 \\
50\end{array}$ \\
\hline D6 & 120 & 24 & 60 & 18 & 18 & 22 & $\begin{array}{c}0 \\
50\end{array}$ \\
\hline
\end{tabular}

\section{RESULTS AND DISCUSSIONS}

\section{Effect of $\mathbf{N}$ and $\mathrm{K}$ and timing of $\mathrm{N}$ application on} wheat yield:

\section{Grain yield:}

Table 3, Figs. 1 and 2 indicated that grain yield of wheat varieties $\mathrm{Giza}_{168}$ and $\mathrm{Sids}_{7}$ was highly significantly increased with increasing $\mathrm{N}$ level up to 120 $\mathrm{Kg} / \mathrm{fed}$.

The highest mean values of grain yield was 21.91 Ardab/ fed., 30.0 ardab / fed. for Giza 168 and Sids 7., respectively. Similar results was reported by ElDesouqi (2000), Muhammad (2001) and Amer (2009). Data Showed that the grain yield of the two wheat varieties significantly increased in the presence of potassium. The highest mean value was recorded under Sids 7 (30.0 ardab / fed.) These results were similar by the data obtained by Genaidy and Hegazay (2001) and Sing and Pathak (2003).Data revealed that time of $\mathrm{N}$ application have a high significant effect on grain yield of the studied varieties. The highest mean values were recorded at $\mathrm{D}_{6}(15: 60: 0: 0)(24: 60: 18: 18)$ for both $\mathrm{N}_{75}$ and $\mathrm{N}_{120}$. Similar results were recorded by El-Desokey et al. (2000) and Warraich et al (2002).

Data indicated that there were high significant effects on grain yield during the two growing seasons due to the interaction between treatments used in the experiments (Table 8).similar results recorded by El Kholy (2000). Data revealed that the highest mean values of grain yield was recorded by $\mathrm{N}_{120} \mathrm{P}_{22} \mathrm{~K}_{50} \mathrm{D}_{6}$ for Giza 168 and Sids 7 (21.91 and 30.0, ardab/fed.), similar results were recorded by Wagan et al (2002) and Koreish et al (2004)

\section{Straw yield:}

Data in Table 4, Figs. 2 and 3 showed that straw yield of the studied wheat varieties were highly significantly increased with increasing $\mathrm{N}$ level up to 120 $\mathrm{Kg} / \mathrm{fed}$. The highest mean values were $5.26,6.0$ tons/fed. for Giza 168 and Sids 7, respectively. Similar results were recorded by El-Sherbieny et al. (1999), and Muhammad (2001).Data revealed that the straw yields of the studied wheat varieties were highly significantly increased in the presence of potassium. The highest mean value was recorded with Sids 7 (6.0 tons/fed.). Similar results recorded by Abd Allah et al (1999), 
Genaidy and Hegazy(2001); Singh and Pathak (2003).Data showed that time of $\mathrm{N}$ application have a high significant effect on straw yield of the studied wheat varieties. The highest mean values were recorded at $\mathrm{D}_{6}$ (15:60:0:0), (24:60:18:18) for both $\mathrm{N}_{75}$ and $\mathrm{N}_{120}$. Ooro and Mwangi (1999), and El Desouqi et al. (2000) reported similar results. Data indicated that there were high significant effects on straw yield due to the interaction between the applied treatments (Table 8).Data showed that the highest mean values of straw yield was recorded at $\mathrm{N}_{120} \mathrm{P}_{22} \mathrm{~K}_{50} \mathrm{D}_{5}$ (15:50:5:5) (5.26 tons/fed. and 6.0 tons/fed for Giza 168 and Sids $\left._{7}\right)$. Similar results were reported by Eisa (1996), El - Banna (2000) and Wagen et al (2002).

Table 3. Effect of $\mathrm{N}, \mathrm{K}$ and timing of $\mathrm{N}$ application on grain yield (ardab/fed.) of Giza 168 and Sids 7 during 2002/2003- 2003/2004 growing seasons

\begin{tabular}{|c|c|c|c|c|c|c|c|c|c|c|c|c|c|}
\hline \multirow{3}{*}{$\begin{array}{c}\text { Growth } \\
\text { stage }\end{array}$} & \multirow{3}{*}{$\begin{array}{l}\text { N-level } \\
\text { ((Kg/fed.) }\end{array}$} & \multicolumn{6}{|c|}{ Var. Giza 168} & \multicolumn{6}{|c|}{ Var. Sids 7} \\
\hline & & \multicolumn{2}{|c|}{$2002 / 2003$} & \multicolumn{2}{|c|}{$2003 / 2004$} & \multicolumn{2}{|c|}{ Mean } & \multicolumn{2}{|c|}{$2002 / 2003$} & \multicolumn{2}{|c|}{$2003 / 2004$} & \multicolumn{2}{|c|}{ Mean } \\
\hline & & $\mathbf{k}_{\mathbf{0}}$ & $K_{50}$ & $\mathbf{k}_{\mathbf{0}}$ & $K_{50}$ & $\mathbf{k}_{\mathbf{0}}$ & $K_{\mathbf{5 0}}$ & $\mathbf{k}_{\mathbf{0}}$ & $\mathbf{K}_{50}$ & $\mathbf{k}_{\mathbf{0}}$ & $\mathbf{K}_{50}$ & $\mathbf{k}_{\mathbf{0}}$ & $\mathbf{K}_{\mathbf{5 0}}$ \\
\hline \multicolumn{2}{|c|}{ Cont. } & $5.81 \mathrm{~d}$ & $5.81 \mathrm{c}$ & $5.12 \mathrm{e}$ & $5.12 \mathrm{c}$ & 5.47 & 5.47 & $6.20 \mathrm{c}$ & $6.2 \mathrm{~d}$ & $5.75 \mathrm{f}$ & $5.75 \mathrm{~g}$ & 5.98 & 5.98 \\
\hline D1 & 75 & $11.17 \mathrm{c}$ & $14.70 \mathrm{~b}$ & $11.21 \mathrm{~d}$ & $14.83 \mathrm{~d}$ & 11.19 & 14.77 & $15.96 \mathrm{~b}$ & $16.94 \mathrm{c}$ & $15.92 \mathrm{e}$ & $18.96 \mathrm{f}$ & 15.94 & 16.95 \\
\hline D2 & 75 & $12.63 \mathrm{c}$ & $16.24 \mathrm{~b}$ & $12.63 \mathrm{c}$ & $16.29 \mathrm{c}$ & 12.63 & 16.27 & $16.5 \mathrm{ab}$ & $17.37 \mathrm{c}$ & $16.52 \mathrm{e}$ & $17.41 \mathrm{f}$ & 16.52 & 17.39 \\
\hline D3 & 75 & $17.26 \mathrm{bc}$ & $19.04 \mathrm{a}$ & $16.6 \mathrm{~b}$ & $19.21 \mathrm{~b}$ & 16.93 & 19.13 & $16.6 \mathrm{ab}$ & $17.89 \mathrm{bc}$ & $16.71 \mathrm{~cd}$ & $17.99 \mathrm{~d}$ & 16.66 & 17.94 \\
\hline D4 & 75 & $17.89 \mathrm{ab}$ & $19.60 \mathrm{a}$ & $18.05 \mathrm{a}$ & $19.73 \mathrm{a}$ & 17.97 & 19.67 & $16.7 \mathrm{ab}$ & $19.32 \mathrm{ab}$ & $16.83 \mathrm{c}$ & $19.41 \mathrm{c}$ & 16.76 & 19.37 \\
\hline D5 & 75 & $17.81 \mathrm{ab}$ & $19.32 \mathrm{a}$ & $18.16 \mathrm{a}$ & $19.75 \mathrm{a}$ & 17.99 & 19.54 & $17.6 \mathrm{ab}$ & $19.6 \mathrm{ab}$ & $17.80 \mathrm{~b}$ & $19.79 \mathrm{~b}$ & 17.72 & 19.70 \\
\hline D6 & 75 & $17.82 \mathrm{a}$ & $19.29 \mathrm{a}$ & $18.09 \mathrm{a}$ & $19.76 \mathrm{a}$ & 17.96 & 19.53 & $18.34 \mathrm{a}$ & $20.16 \mathrm{a}$ & $5 \mathrm{a}$ & $20.19 \mathrm{a}$ & 18.40 & 20.18 \\
\hline D1 & 120 & $16.22 \mathrm{~b}$ & $16.80 \mathrm{bc}$ & $15.63 \mathrm{e}$ & $16.85 \mathrm{e}$ & 16.43 & 16.83 & $18.4 \mathrm{c}$ & $25.90 \mathrm{~d}$ & $20.64 \mathrm{~d}$ & $25.89 \mathrm{f}$ & 19.52 & 25.89 \\
\hline D2 & 120 & $17.10 \mathrm{ab}$ & $17.51 \mathrm{~b}$ & $17.12 \mathrm{~d}$ & $17.52 \mathrm{~d}$ & 17.11 & 17.15 & $22.4 \mathrm{~b}$ & $26.77 \mathrm{~cd}$ & & $26.82 \mathrm{e}$ & 22.41 & 26.79 \\
\hline D3 & 120 & $17.92 \mathrm{ab}$ & $19.43 \mathrm{a}$ & $18.15 \mathrm{~b}$ & $19.75 \mathrm{~b}$ & 18.04 & 19.59 & $23.49 \mathrm{a}$ & $28.0 \mathrm{bc}$ & $23.53 \mathrm{~b}$ & $28.11 \mathrm{~d}$ & 23.51 & 28.05 \\
\hline D4 & 120 & $18.34 \mathrm{a}$ & $20.46 \mathrm{a}$ & $18.61 \mathrm{a}$ & $20.98 \mathrm{a}$ & 18.48 & 20.72 & $25.06 \mathrm{a}$ & $28.26 \mathrm{ab}$ & $23.13 \mathrm{a}$ & $28.69 \mathrm{c}$ & 25.09 & 28.62 \\
\hline D5 & 120 & $19.04 \mathrm{a}$ & $20.85 \mathrm{a}$ & $19.25 \mathrm{a}$ & $21.98 \mathrm{a}$ & 19.15 & 21.42 & $25.17 \mathrm{a}$ & $29.12 \mathrm{ab}$ & $25.29 \mathrm{a}$ & $29.19 \mathrm{~b}$ & 25.23 & 29.15 \\
\hline D6 & 120 & $18.76 \mathrm{a}$ & $21.0 \mathrm{a}$ & $19.26 \mathrm{a}$ & $21.91 \mathrm{a}$ & 19.01 & 21.46 & $25.20 \mathrm{a}$ & $30.37 \mathrm{a}$ & $25.32 \mathrm{a}$ & $30.0 \mathrm{a}$ & 25.26 & 30.18 \\
\hline
\end{tabular}

Where LSD for 2 D-means at each N x K x V equal (0.66)at 0.05 and (0.90) at $0.01(2002 / 2003)$ and (0. 25) at 0.05 and (0.34) at 0.01 (2003/2004).

Table 4. Effect of $N$, $K$ and timing of $N$ application on straw yield ( $t / f e d$.$) of Giza 168$ and Sids 7 during 2002/2003 - 2003/2004 growing seasons

\begin{tabular}{|c|c|c|c|c|c|c|c|c|c|c|c|c|c|}
\hline \multirow{3}{*}{$\begin{array}{c}\text { Growth } \\
\text { stage }\end{array}$} & \multirow{3}{*}{$\begin{array}{c}\text { N level } \\
\text { (Kg/fed) }\end{array}$} & \multicolumn{6}{|c|}{ Var. Giza 168} & \multicolumn{6}{|c|}{ Var. Sids 7} \\
\hline & & \multicolumn{2}{|c|}{$2002 / 2003$} & \multicolumn{2}{|c|}{$2003 / 2004$} & \multicolumn{2}{|c|}{ Mean } & \multicolumn{2}{|c|}{$2002 / 2003$} & \multicolumn{2}{|c|}{$2003 / 2004$} & \multicolumn{2}{|c|}{ Mean } \\
\hline & & $\mathbf{k}_{\mathbf{0}}$ & $K_{50}$ & $\mathbf{k}_{\mathbf{0}}$ & $K_{50}$ & $\mathbf{k}_{\mathbf{0}}$ & $K_{50}$ & $\mathbf{k}_{\mathbf{0}}$ & $K_{50}$ & $\mathbf{k}_{\mathbf{0}}$ & $K_{50}$ & $\mathbf{k}_{\mathbf{0}}$ & $K_{50}$ \\
\hline \multicolumn{2}{|c|}{ Cont. } & $1.75 \mathrm{c}$ & $1.75 \mathrm{c}$ & $1.65 \mathrm{c}$ & $1.65 \mathrm{e}$ & 1.7 & 1.7 & $1.95 \mathrm{c}$ & $1.95 \mathrm{c}$ & $1.92 \mathrm{~d}$ & $1.92 \mathrm{e}$ & 1.93 & 1.93 \\
\hline D1 & 75 & $3.15 \mathrm{~b}$ & $3.65 \mathrm{~b}$ & $3.20 \mathrm{~b}$ & 3.68 & 3.17 & 3.66 & $4.0 \mathrm{~b}$ & $4.0 \mathrm{~b}$ & $4.10 \mathrm{c}$ & $4.12 \mathrm{~d}$ & 4.0 & 4.06 \\
\hline D2 & 75 & $3.20 \mathrm{~b}$ & $3.70 \mathrm{~b}$ & $3.22 \mathrm{~b}$ & $3.75 \mathrm{c}$ & 3.21 & 3.73 & $4.01 \mathrm{a}$ & $4.22 \mathrm{~b}$ & $4.10 \mathrm{c}$ & $4.32 \mathrm{~d}$ & 5.05 & 4.27 \\
\hline D3 & 75 & $3.28 \mathrm{~b}$ & $3.78 \mathrm{~b}$ & $3.78 \mathrm{a}$ & $3.80 \mathrm{c}$ & 3.53 & 3.79 & $4.0 \mathrm{~b}$ & $4.30 \mathrm{a}$ & $4.1 \mathrm{c}$ & $4.38 \mathrm{~b}$ & 4.0 & 4.34 \\
\hline D4 & 75 & $3.31 \mathrm{a}$ & $3.81 \mathrm{a}$ & $3.79 \mathrm{a}$ & $3.91 \mathrm{~b}$ & 3.55 & 3.86 & $4.10 \mathrm{a}$ & $4.35 \mathrm{a}$ & $4.21 \mathrm{~b}$ & $4.40 \mathrm{ab}$ & 4.15 & 4.37 \\
\hline D5 & 75 & $3.41 \mathrm{a}$ & $3.95 \mathrm{a}$ & $3.80 \mathrm{a}$ & $3.98 \mathrm{a}$ & 3.61 & 3.97 & $4.18 \mathrm{a}$ & $4.36 \mathrm{a}$ & $4.20 \mathrm{~b}$ & $4.4 \mathrm{ab}$ & 4.19 & 4.38 \\
\hline D6 & 75 & $3.68 \mathrm{a}$ & $3.98 \mathrm{a}$ & $3.79 \mathrm{a}$ & $3.90 \mathrm{a}$ & 3.74 & 3.94 & $4.25 \mathrm{a}$ & $4.40 \mathrm{a}$ & $4.27 \mathrm{a}$ & $4.45 \mathrm{a}$ & 4.26 & 4.47 \\
\hline D1 & 120 & $4.11 \mathrm{c}$ & $4.51 \mathrm{c}$ & $4.21 \mathrm{e}$ & $4.61 \mathrm{f}$ & 4.16 & 4.56 & $4.65 \mathrm{c}$ & $5.17 \mathrm{c}$ & $4.67 \mathrm{~d}$ & $5.27 \mathrm{f}$ & 4.66 & 5.22 \\
\hline D2 & 120 & $4.20 \mathrm{~b}$ & $4.61 \mathrm{~b}$ & $4.30 \mathrm{~d}$ & $4.70 \mathrm{e}$ & 4.25 & 4.66 & $4.65 \mathrm{c}$ & $5.32 \mathrm{c}$ & $4.70 \mathrm{~d}$ & $5.39 \mathrm{e}$ & 4.67 & 5.35 \\
\hline D3 & 120 & $4.31 \mathrm{~b}$ & $4.90 \mathrm{~b}$ & $4.41 \mathrm{c}$ & $4.92 \mathrm{~d}$ & 4.36 & 4.90 & $4.70 \mathrm{~b}$ & $5.60 \mathrm{~b}$ & $4.80 \mathrm{c}$ & $5.69 \mathrm{~d}$ & 4.75 & 5.64 \\
\hline D4 & 120 & $4.83 \mathrm{a}$ & $5.0 \mathrm{a}$ & $4.86 \mathrm{~b}$ & $5.0 \mathrm{c}$ & 4.84 & 5.0 & $4.95 \mathrm{a}$ & $5.82 \mathrm{a}$ & $4.96 \mathrm{~b}$ & $5.90 \mathrm{c}$ & 4.95 & 5.86 \\
\hline D5 & 120 & $4.98 \mathrm{a}$ & $5.25 \mathrm{a}$ & $4.97 \mathrm{a}$ & $5.28 \mathrm{a}$ & 4.97 & 5.26 & $5.08 \mathrm{a}$ & $5.95 \mathrm{a}$ & $5.0 \mathrm{~b}$ & $5.96 \mathrm{~b}$ & 5.0 & 5.95 \\
\hline D6 & 120 & $4.95 \mathrm{a}$ & $5.19 \mathrm{a}$ & $4.95 \mathrm{a}$ & $5.20 \mathrm{~b}$ & 4.95 & 5.19 & $5.10 \mathrm{a}$ & $6.00 \mathrm{a}$ & $5.20 \mathrm{a}$ & $6.10 \mathrm{a}$ & 5.15 & 6.0 \\
\hline
\end{tabular}

Where LSD for 2 D-means at eatch N x K x V equal (0.35)at 0.05 and (0.48) at $0.01(2002 / 2003)$ and $(0.32)$ at 0.05 and $(0.44)$ at 0.01 $(2003 / 2004)$ 


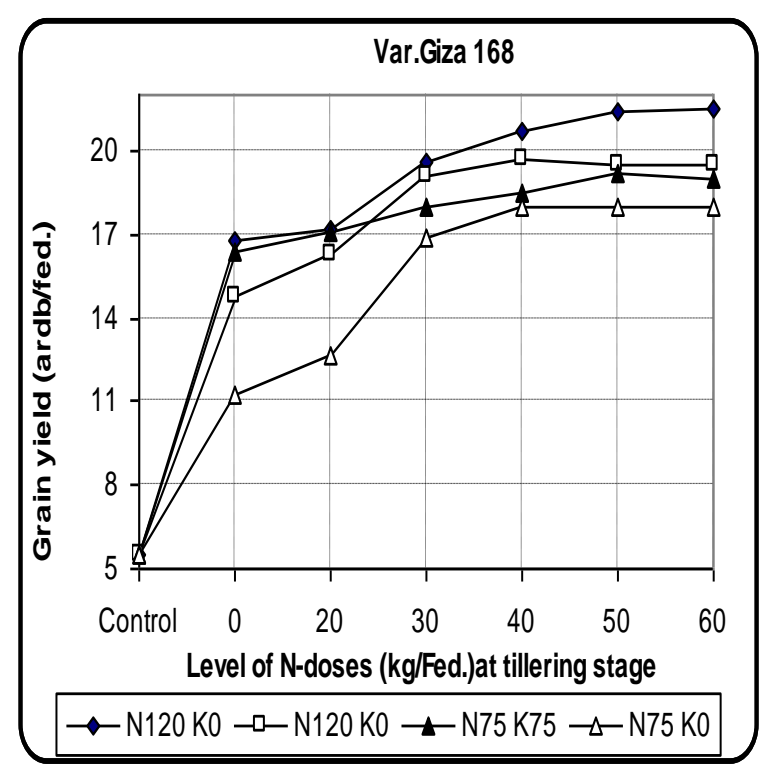

Fig. 1. Grain yield of wheat Giza 168 as affected by $\mathrm{N}, \mathrm{K}$ and timing of $\mathrm{N}$ application

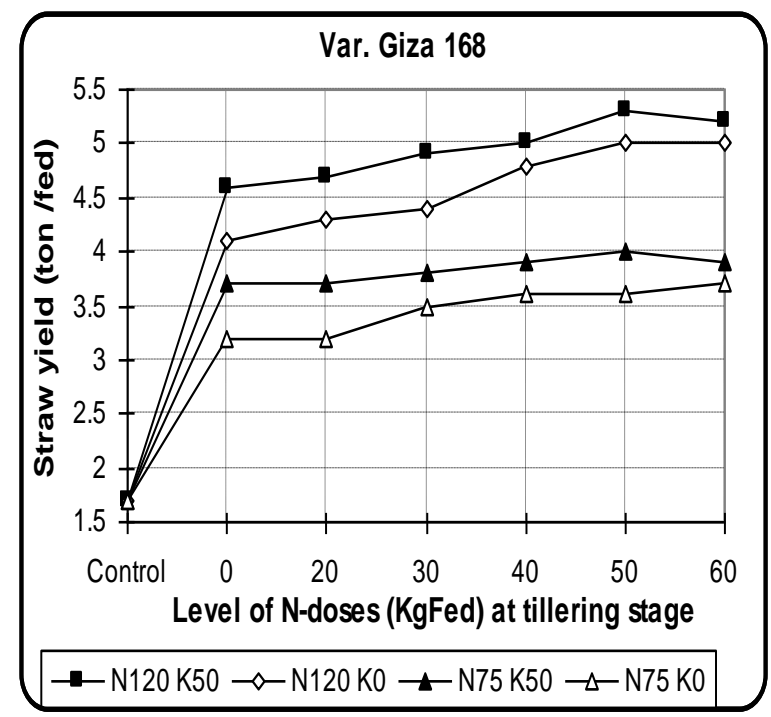

Fig. 3. Straw yield of wheat Giza 168 as affected by $N, K$ and timing of $N$ application

Number of spikes $/ \mathbf{m}^{2}$ :

Table 5 showed that the number of spikes $/ \mathrm{m}^{2}$ of the two wheat varieties was highly significantly increased by increasing $\mathrm{N}$ level up to $120 \mathrm{Kg} / \mathrm{fed}$. The highest mean values were 436 and 300 for Giza 168 and Sids 7 . Similar results were reported by Sabry et al. (1999) and Amer (2009). Data showed that the number of spikes $/ \mathrm{m}^{2}$ of the studied wheat varieties were significantly increased in the presence of potassium and the highest

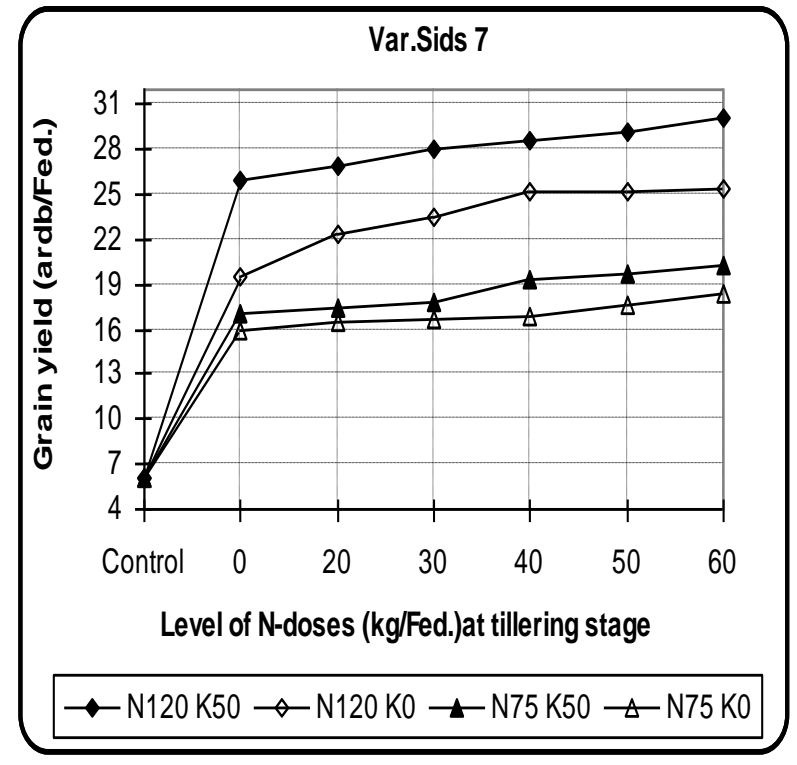

Fig. 2. Grain yield of wheat Sids 7 as affected by $\mathrm{N}, \mathrm{K}$ and timing of $\mathrm{N}$ application

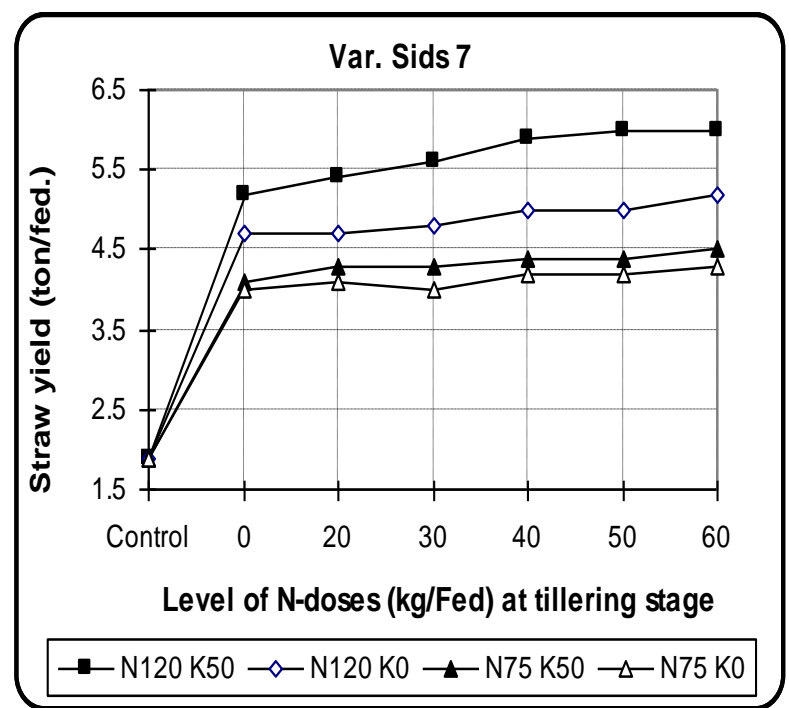

Fig. 4. Straw yield of wheat Sids 7 as affected by $\mathrm{N}, \mathrm{K}$ and timing of $\mathrm{N}$ application

mean values was recorded with Giza 168 (436) similar results were reported by Singh and Pathak (2003).

Data revealed that time of $\mathrm{N}$ application have high significant effect on number of spikes $/ \mathrm{m}^{2}$ of the studied wheat varieties. The highest mean values were recorded at $\mathrm{D}_{6}(15: 60: 0: 0)(24: 60: 18: 18)$ for $\mathrm{N}_{75}$ and $\mathrm{N}_{120}$, similar results were reported by El far and El - Nagar (1995), Rahman et al. (2002) and Warraich et al. (2002).Data indicated that there were high significant effects on 
number of spikes $/ \mathrm{m}^{2}$-due to the interaction between treatments (Table 8).

\section{Number of grains/spike:}

Data in Table 6 showed that the number of grains per spike of the studied wheat varieties was highly significantly increased by increasing $\mathrm{N}$ level up to 120 $\mathrm{Kg} /$ feddan. The highest mean values were 83 and 104 for Giza 168 and Sids 7. Similar results were reported by Abd El Wahed (1997), Maqsood et al (2002) and Muhammed (2001). Data showed that, number of grains per spike of the studied wheat varieties was highly significantly increased in the presence of potassium and the highest mean value was recorded under Sids 7 . This result was similarly observed by Abd Allah et al. (1999).Data showed that time of $\mathrm{N}$ application have high significant effect on number of grains per spike of the studied wheat varieties. The highest mean values were recorded at $D_{6}$ (15:60:0:0) (24:60:18:18) for both $\mathrm{N}_{75}$ and $\mathrm{N}_{120}$ This result was similarly observed by Faizy et al. (1986 b) and Ewart and Honermeier (1999).Data indicated that there were high significant effects on number of grains /spike due to the interaction between treatments (Table 8 ). This result was similarly observed by El Kholy (2000).

Table 5. Effect of $\mathrm{N}, \mathrm{K}$ and timing of $\mathrm{N}$ application on number of spikes $/ \mathrm{m} 2$ of Giza 168 and Sids 7 during 2002/2003- 2003/2004 growing seasons

\begin{tabular}{|c|c|c|c|c|c|c|c|c|c|c|c|c|c|}
\hline \multirow{3}{*}{$\begin{array}{c}\text { Growth } \\
\text { stage }\end{array}$} & \multirow{3}{*}{$\begin{array}{c}\text { N- level } \\
\text { (Kg/fed) }\end{array}$} & \multicolumn{6}{|c|}{ Var. Giza 168} & \multicolumn{6}{|c|}{ Var. Sids 7} \\
\hline & & \multicolumn{2}{|c|}{$2002 / 2003$} & \multicolumn{2}{|c|}{$2003 / 2004$} & \multicolumn{2}{|c|}{ Mean } & \multicolumn{2}{|c|}{$2002 / 2003$} & \multicolumn{2}{|c|}{$2003 / 2004$} & \multicolumn{2}{|c|}{ Mean } \\
\hline & & $\mathbf{k}_{\mathbf{0}}$ & $K_{50}$ & $\mathbf{k}_{\mathbf{0}}$ & $K_{50}$ & $\mathbf{k}_{\mathbf{0}}$ & $K_{50}$ & $\mathbf{k}_{\mathbf{0}}$ & $\mathbf{K}_{\mathbf{5 0}}$ & $\mathbf{k}_{\mathbf{0}}$ & $K_{50}$ & $\mathbf{k}_{\mathbf{0}}$ & $K_{50}$ \\
\hline \multicolumn{2}{|c|}{ Cont. } & $186.5 \mathrm{f}$ & $186.5 \mathrm{~g}$ & $180 \mathrm{~g}$ & $180 \mathrm{~g}$ & 183.3 & 183.3 & $180 \mathrm{e}$ & $180 \mathrm{~d}$ & $175 \mathrm{~g}$ & $175 \mathrm{f}$ & 177.5 & 177.5 \\
\hline D1 & 75 & $260 \mathrm{e}$ & $290 \mathrm{f}$ & $262 \mathrm{f}$ & $292 \mathrm{f}$ & 261 & 291 & $200 \mathrm{~d}$ & $200 \mathrm{c}$ & $201 \mathrm{f}$ & $201 \mathrm{e}$ & 200.5 & 200.5 \\
\hline D2 & 75 & $290 \mathrm{~d}$ & $310 \mathrm{e}$ & $292 \mathrm{e}$ & $312 \mathrm{f}$ & 291 & 311 & $210 \mathrm{~cd}$ & $240 \mathrm{~b}$ & $211 \mathrm{e}$ & $242 \mathrm{~d}$ & 210.5 & 241 \\
\hline D3 & 75 & $300 \mathrm{~cd}$ & $330 \mathrm{~d}$ & $313 \mathrm{~d}$ & $332 \mathrm{~d}$ & 306.5 & 331 & $220 \mathrm{c}$ & $250 \mathrm{ab}$ & $226 \mathrm{~d}$ & $253 \mathrm{c}$ & 223 & 251.5 \\
\hline D4 & 75 & $319 \mathrm{~b}$ & $350 \mathrm{c}$ & $321 \mathrm{c}$ & $353 \mathrm{c}$ & 320 & 351.5 & $235 \mathrm{~b}$ & $258 \mathrm{a}$ & $238 \mathrm{c}$ & $259 \mathrm{~b}$ & 236.5 & 258.5 \\
\hline D5 & 75 & $314 \mathrm{bc}$ & $365 \mathrm{~b}$ & $339 \mathrm{~b}$ & $374 \mathrm{~b}$ & 326.5 & 369.5 & $240 \mathrm{~b}$ & $260 \mathrm{a}$ & $242 \mathrm{~b}$ & $262 \mathrm{a}$ & 241 & 261 \\
\hline D6 & 75 & $350 \mathrm{a}$ & $400 \mathrm{a}$ & $352 \mathrm{a}$ & $390 \mathrm{a}$ & 351 & 395 & $259 \mathrm{a}$ & $260 \mathrm{a}$ & $260 \mathrm{a}$ & $263 \mathrm{a}$ & 259.5 & 261.5 \\
\hline D1 & 120 & $300 \mathrm{e}$ & $300 \mathrm{~d}$ & $302 \mathrm{f}$ & $311 \mathrm{f}$ & 301 & 306.5 & $210 \mathrm{~d}$ & $250 \mathrm{~d}$ & $211 \mathrm{f}$ & $253 \mathrm{~d}$ & 210.5 & 251.5 \\
\hline D2 & 120 & $316.75 \mathrm{de}$ & $345 \mathrm{c}$ & $313 \mathrm{e}$ & $347 \mathrm{e}$ & 314.9 & 346 & $225 \mathrm{c}$ & $275 \mathrm{c}$ & $228 \mathrm{e}$ & $278 \mathrm{c}$ & 226.5 & 276.5 \\
\hline D3 & 120 & $320 \mathrm{~d}$ & $355.75 \mathrm{c}$ & $321 \mathrm{~d}$ & $357 \mathrm{~d}$ & 320.5 & 356.4 & $235 \mathrm{c}$ & $285 \mathrm{~b}$ & $235 \mathrm{~d}$ & $287 \mathrm{~b}$ & 235 & 286 \\
\hline D4 & 120 & $346 \mathrm{c}$ & $380 \mathrm{~b}$ & $347 \mathrm{c}$ & $382 \mathrm{c}$ & 346.5 & 381 & $250 \mathrm{~b}$ & $286.75 \mathrm{~b}$ & $252 \mathrm{c}$ & $289 \mathrm{~b}$ & 251 & 287.9 \\
\hline D5 & 120 & $380 \mathrm{~b}$ & $450 \mathrm{a}$ & $384 \mathrm{~b}$ & $435 \mathrm{a}$ & 382 & 442.5 & $260 \mathrm{~b}$ & $289.75 \mathrm{a}$ & $263 \mathrm{~b}$ & $291 \mathrm{a}$ & 261.5 & 290.4 \\
\hline D6 & 120 & $400 \mathrm{a}$ & $440.75 \mathrm{a}$ & $400 \mathrm{a}$ & $431 \mathrm{~b}$ & 400 & 435.9 & $285 \mathrm{a}$ & $299 \mathrm{a}$ & $298 \mathrm{a}$ & $301 \mathrm{a}$ & 291.5 & 300 \\
\hline
\end{tabular}

Where LSD for 2 D-means at each N x K x V equal (6.96)at 0.05 and (9.55) at 0.01 (2002/2003) and (0.53) at 0.05 and (0.72) at 0.01 (2003/2004)

Table 6. Effect of $\mathrm{N}, \mathrm{K}$ and timing of $\mathrm{N}$ application on number of grains per spike of Giza 168 and Sids 7 during 2002/2003 - 2003/2004 growing seasons

\begin{tabular}{|c|c|c|c|c|c|c|c|c|c|c|c|c|c|}
\hline \multirow{3}{*}{$\begin{array}{c}\text { Jrowth } \\
\text { stage }\end{array}$} & \multirow{3}{*}{$\begin{array}{l}\text { N level } \\
\text { (Kg/fed) }\end{array}$} & \multicolumn{6}{|c|}{ Var. Giza 168} & \multicolumn{6}{|c|}{ Var. Sids 7} \\
\hline & & \multicolumn{2}{|c|}{$2002 / 2003$} & \multicolumn{2}{|c|}{$2003 / 2004$} & \multicolumn{2}{|c|}{ Mean } & \multicolumn{2}{|c|}{$2002 / 2003$} & \multicolumn{2}{|c|}{$2003 / 2004$} & \multicolumn{2}{|c|}{ Mean } \\
\hline & & $\mathbf{k}_{\mathbf{0}}$ & $K_{50}$ & $\mathbf{k}_{\mathbf{0}}$ & $\mathbf{K}_{\mathbf{5 0}}$ & $\mathbf{k}_{\mathbf{0}}$ & $K_{50}$ & $\mathbf{k}_{\mathbf{0}}$ & $K_{50}$ & $\mathbf{k}_{\mathbf{0}}$ & $K_{50}$ & $\mathbf{k}_{\mathbf{0}}$ & $K_{50}$ \\
\hline \multicolumn{2}{|c|}{ Cont. } & $42.5 \mathrm{~d}$ & $42.5 \mathrm{~d}$ & $36 \mathrm{e}$ & $36 \mathrm{~d}$ & 39.25 & 39.25 & $59.5 \mathrm{e}$ & $59.5 \mathrm{e}$ & $49 \mathrm{f}$ & $49 \mathrm{e}$ & 54.3 & 54.3 \\
\hline D1 & 75 & $68 \mathrm{c}$ & $69 \mathrm{c}$ & $69 \mathrm{~d}$ & $71 \mathrm{c}$ & 68.50 & 70 & $81 \mathrm{~d}$ & $89 \mathrm{~d}$ & $82 \mathrm{e}$ & $89 \mathrm{~d}$ & 81.5 & 89 \\
\hline D2 & 75 & $72.5 \mathrm{~b}$ & $75 \mathrm{~b}$ & $70 \mathrm{c}$ & $72 \mathrm{~b}$ & 71.3 & 73.5 & $84.75 \mathrm{c}$ & $91.25 \mathrm{~cd}$ & $85 \mathrm{~d}$ & $92 \mathrm{c}$ & 84.9 & 91.7 \\
\hline D3 & 75 & $73 \mathrm{a}$ & $75.75 \mathrm{a}$ & $72 \mathrm{~b}$ & $74 \mathrm{~b}$ & 72.5 & 74.9 & $84.5 \mathrm{c}$ & $92.25 \mathrm{bc}$ & $86 \mathrm{~d}$ & $93.5 \mathrm{~b}$ & 85.3 & 92.9 \\
\hline D4 & 75 & $73 \mathrm{a}$ & $76.5 \mathrm{a}$ & $73 \mathrm{~b}$ & $74 \mathrm{~b}$ & 73 & 75.3 & $87.75 \mathrm{~b}$ & $91.8 \mathrm{bcd}$ & $88 \mathrm{c}$ & $94.5 \mathrm{~b}$ & 87.9 & 93.12 \\
\hline D5 & 75 & $73.75 \mathrm{a}$ & $76 \mathrm{a}$ & $75 \mathrm{a}$ & $76 \mathrm{a}$ & 74.4 & 76 & $92 \mathrm{a}$ & $94.75 \mathrm{ab}$ & $92 \mathrm{~b}$ & $96 \mathrm{a}$ & 92 & 95.4 \\
\hline D6 & 75 & $74 \mathrm{a}$ & $77 \mathrm{a}$ & $75 a$ & $77 \mathrm{a}$ & 74.5 & 77 & $93 \mathrm{a}$ & $96.75 \mathrm{a}$ & $94 \mathrm{a}$ & $97 \mathrm{a}$ & 93.5 & 96.9 \\
\hline D1 & 120 & $73.75 \mathrm{~b}$ & $77 \mathrm{c}$ & $74 \mathrm{c}$ & $75 \mathrm{~d}$ & 73.9 & 76 & $85.75 \mathrm{c}$ & $98.5 \mathrm{bc}$ & $86 \mathrm{e}$ & $96 \mathrm{~d}$ & 85.88 & 97.3 \\
\hline D2 & 120 & $76.25 \mathrm{~b}$ & $78 \mathrm{c}$ & $75 \mathrm{~b}$ & $76 \mathrm{c}$ & 75.6 & 77 & $85.5 \mathrm{c}$ & $96.75 \mathrm{c}$ & $88 \mathrm{~d}$ & $98 \mathrm{c}$ & 86.8 & 97.4 \\
\hline D3 & 120 & $77 \mathrm{ab}$ & $79 \mathrm{~b}$ & $76 a$ & $79 \mathrm{~b}$ & 76.5 & 79 & $88.25 \mathrm{c}$ & 99 bc & $94 \mathrm{c}$ & $99 \mathrm{~b}$ & 94.03 & 99 \\
\hline D4 & 120 & $78 \mathrm{a}$ & $80 \mathrm{~b}$ & $78 \mathrm{a}$ & $80 \mathrm{a}$ & 78 & 80 & $94.06 \mathrm{~b}$ & $99 \mathrm{bc}$ & $95.75 \mathrm{~b}$ & $100 \mathrm{~b}$ & 92 & 99.5 \\
\hline D5 & 120 & $78 \mathrm{a}$ & $83 \mathrm{~b}$ & $79 a$ & $81 \mathrm{a}$ & 78.5 & 82 & $94 \mathrm{~b}$ & $100.3 \mathrm{ab}$ & $96 \mathrm{~b}$ & $104.5 \mathrm{a}$ & 95 & 102.4 \\
\hline D6 & 120 & $79 a$ & $84 \mathrm{a}$ & $79 \mathrm{a}$ & $82 a$ & 79 & 83 & $99 \mathrm{a}$ & $102.75 \mathrm{a}$ & $100 \mathrm{a}$ & $104.3 \mathrm{a}$ & 99.5 & 103.5 \\
\hline
\end{tabular}


Table 7. Effect of $N$, $K$ and timing of $N$ application on 1000- grain weight(g) of Giza 168 and Sids 7 during 2002/2003 - 2003/2004 growing seasons

\begin{tabular}{|c|c|c|c|c|c|c|c|c|c|c|c|c|c|}
\hline \multirow{3}{*}{$\begin{array}{c}\text { Growth } \\
\text { stage }\end{array}$} & \multirow{3}{*}{$\begin{array}{l}\text { N level } \\
\text { (Kg/fed) }\end{array}$} & \multicolumn{6}{|c|}{ Var. Giza 168} & \multicolumn{6}{|c|}{ Var. Sids 7} \\
\hline & & \multicolumn{2}{|c|}{$2002 / 2003$} & \multicolumn{2}{|c|}{$2003 / 2004$} & \multicolumn{2}{|c|}{ Mean } & \multicolumn{2}{|c|}{$2002 / 2003$} & \multicolumn{2}{|c|}{$2003 / 2004$} & \multicolumn{2}{|c|}{ Mean } \\
\hline & & $\mathbf{k}_{\mathbf{0}}$ & $K_{50}$ & $\mathbf{k}_{\mathbf{0}}$ & $K_{50}$ & $\mathbf{k}_{\mathbf{0}}$ & $\mathbf{K}_{\mathbf{5 0}}$ & $\mathbf{k}_{\mathbf{0}}$ & $K_{50}$ & $\mathbf{k}_{\mathbf{0}}$ & $K_{50}$ & $\mathbf{k}_{\mathbf{0}}$ & $K_{50}$ \\
\hline \multicolumn{2}{|c|}{ Cont. } & $25.0 \mathrm{e}$ & $25.0 \mathrm{e}$ & $25.50 \mathrm{e}$ & $25.50 \mathrm{c}$ & 25.25 & 25.25 & $32.50 \mathrm{~d}$ & $32.50 \mathrm{e}$ & $32.0 \mathrm{c}$ & $32.0 \mathrm{e}$ & 32.25 & 32.25 \\
\hline D1 & 75 & $37.0 \mathrm{~b}$ & $37.60 \mathrm{~b}$ & $37.50 \mathrm{~b}$ & $37.80 \mathrm{a}$ & 37.25 & 37.65 & $53.0 \mathrm{bc}$ & $55.10 \mathrm{c}$ & $53.31 \mathrm{~b}$ & $55.30 \mathrm{~d}$ & 53.15 & 55.2 \\
\hline D2 & 75 & $37.1 \mathrm{~b}$ & $37.60 \mathrm{~b}$ & $37.80 \mathrm{~b}$ & $37.80 \mathrm{a}$ & 37.5 & 37.7 & $53.20 \mathrm{~b}$ & $56.20 \mathrm{~b}$ & $53.41 \mathrm{~b}$ & $56.50 \mathrm{~b}$ & 53.37 & 56.35 \\
\hline D3 & 75 & $38.0 \mathrm{a}$ & $38.50 \mathrm{a}$ & $38.0 \mathrm{a}$ & $38.11 \mathrm{a}$ & 38.0 & 38.31 & $53.0 \mathrm{bc}$ & $56.30 \mathrm{~b}$ & $53.20 \mathrm{~b}$ & $56.50 \mathrm{~b}$ & 53.1 & 56.40 \\
\hline D4 & 75 & $36.0 \mathrm{c}$ & $36.30 \mathrm{c}$ & $37.0 \mathrm{c}$ & $37.15 \mathrm{~b}$ & 36.50 & 36.73 & $54.50 \mathrm{a}$ & $57.0 \mathrm{a}$ & $54.0 \mathrm{a}$ & $57.0 \mathrm{a}$ & 54.25 & 57 \\
\hline D5 & 75 & $35.0 \mathrm{~d}$ & $35.37 \mathrm{~d}$ & $36.11 \mathrm{~d}$ & $37.10 \mathrm{~b}$ & 35.56 & 36.21 & $52.50 \mathrm{c}$ & $55.0 \mathrm{~d}$ & $53.31 \mathrm{~b}$ & $56.0 \mathrm{c}$ & 52.41 & 55.5 \\
\hline D6 & 75 & $35.10 \mathrm{~d}$ & $35.53 \mathrm{~d}$ & $36.19 \mathrm{~d}$ & $36.88 \mathrm{~b}$ & 35.65 & 36.21 & $52.60 \mathrm{bc}$ & $55.0 \mathrm{~d}$ & $53.21 \mathrm{~b}$ & $55.30 \mathrm{~d}$ & 52.91 & 55.15 \\
\hline D1 & 120 & $37.6 \mathrm{a}$ & $37.7 \mathrm{~b}$ & $37.5 \mathrm{a}$ & $37.9 \mathrm{~d}$ & 37.55 & 37.8 & $54.5 \mathrm{c}$ & $61.1 \mathrm{c}$ & $54.6 \mathrm{~b}$ & $61.5 \mathrm{c}$ & 54.55 & 61.3 \\
\hline D2 & 120 & $36.25 \mathrm{~b}$ & $38.1 \mathrm{~b}$ & $36.6 \mathrm{~b}$ & $38.0 \mathrm{~d}$ & 36.42 & 38.05 & $54.9 \mathrm{c}$ & $59.8 \mathrm{~d}$ & $54.81 \mathrm{~b}$ & $60.50 \mathrm{e}$ & 54.86 & 60.15 \\
\hline D3 & 120 & $36.0 \mathrm{a}$ & $40.15 \mathrm{a}$ & $36.4 \mathrm{~b}$ & $40.75 \mathrm{a}$ & 36.20 & 40.45 & $56.8 \mathrm{a}$ & $65.6 \mathrm{a}$ & $55.91 \mathrm{a}$ & $62.9 \mathrm{a}$ & 56.36 & 62.75 \\
\hline D4 & 120 & $35.50 \mathrm{~d}$ & $38.9 \mathrm{c}$ & $36.03 \mathrm{c}$ & $39.48 \mathrm{c}$ & 35.76 & 39.19 & $56.1 \mathrm{~b}$ & $61.75 \mathrm{~b}$ & $56.08 \mathrm{a}$ & $62.41 \mathrm{~b}$ & 56.09 & 62.08 \\
\hline D5 & 120 & $35.40 \mathrm{~d}$ & $35.9 \mathrm{c}$ & $35.66 \mathrm{~d}$ & $40.11 \mathrm{~b}$ & 35.53 & 38 & $56.35 \mathrm{~b}$ & $59.70 \mathrm{~d}$ & $56.10 \mathrm{a}$ & $60.97 \mathrm{~d}$ & 56.23 & 60.34 \\
\hline D6 & 120 & $35.0 \mathrm{bc}$ & $35.9 \mathrm{~d}$ & $35.9 \mathrm{~cd}$ & $38.10 \mathrm{~d}$ & 35.45 & 37 & $56.35 \mathrm{~b}$ & $61.0 \mathrm{c}$ & 55.81a & $61.05 \mathrm{~d}$ & 56.08 & 61.02 \\
\hline
\end{tabular}
Where LSD for 2 D-means at each N x K x V equal (0.83)at 0.05 and (1.14) at $0.01(2002 / 2003)$ and $(0.21)$ at 0.05 and $(0.29)$ at 0.01 (2003/2004).

Table 8. The main effect of $\mathrm{N}, \mathrm{K}$ and timing of $\mathrm{N}$ application and its interactions on yield and some components

\begin{tabular}{|c|c|c|c|c|c|c|c|c|c|c|}
\hline \multirow[t]{2}{*}{ Treatment } & \multicolumn{2}{|c|}{$\begin{array}{l}\text { Grain yield } \\
\text { ardab/fed }\end{array}$} & \multicolumn{2}{|c|}{$\begin{array}{c}\text { Straw yield } \\
\text { ton/fed }\end{array}$} & \multicolumn{2}{|c|}{$\begin{array}{c}1000 \text { grain } \\
\text { weight(g) }\end{array}$} & \multicolumn{2}{|c|}{ No. of spikes / $\mathbf{m}^{2}$} & \multicolumn{2}{|c|}{$\begin{array}{l}\text { No of grains } \\
\text { /spike }\end{array}$} \\
\hline & 2003 & 2004 & 2003 & 2004 & 2003 & 2004 & 2003 & 2004 & 2003 & 2004 \\
\hline Cont. & 6.005 & 5.5 & 1.85 & 1.78 & 28.87 & 28.75 & 183.13 & 177.5 & 51.0 & 42.5 \\
\hline D1 & 17.013 & 17.24 & 4.15 & 4.23 & 46.72 & 46.92 & 251.25 & 254.13 & 80.82 & 80.25 \\
\hline D2 & 18.319 & 18.34 & 4.23 & 4.31 & 46.65 & 46.90 & 275.72 & 277.88 & 82.50 & 82.0 \\
\hline D3 & 19.954 & 20.007 & 4.35 & 4.48 & 48.17 & 47.72 & 286.97 & 290.50 & 82.75 & 84.19 \\
\hline D4 & 20.74 & 20.92 & 4.52 & 4.62 & 47.02 & 47.93 & 303.09 & 305.13 & 84.21 & 85.41 \\
\hline D5 & 21.070 & 21.40 & 4.64 & 4.69 & 45.67 & 46.92 & 307.34 & 323.75 & 86.53 & 87.44 \\
\hline D6 & 20.689 & 21.62 & 4.69 & 4.73 & 45.95 & 46.56 & 348.22 & 333.88 & 88.19 & 88.53 \\
\hline F. test & $* *$ & $* *$ & $* *$ & $* *$ & $* *$ & $* *$ & $* *$ & $* *$ & $* *$ & $* *$ \\
\hline LSD $_{0.05}$ & 0.66 & 0.25 & 0.35 & 0.32 & 0.83 & 0.21 & 6.96 & 0.53 & 1.45 & 0.62 \\
\hline LSD $_{0.01}$ & 0.90 & 0.34 & 0.48 & 0.44 & 1.14 & 0.29 & 9.55 & 0.72 & 1.99 & 0.85 \\
\hline $\mathbf{N}_{75}$ & 15.709 & 15.71 & 3.58 & 3.67 & 43.21 & 43.55 & 262.25 & 267.86 & 77.36 & 76.30 \\
\hline $\mathbf{N}_{120}$ & 19.662 & 20.01 & 4.54 & 4.57 & 45.09 & 45.35 & 296.53 & 292.93 & 81.60 & 80.91 \\
\hline F. test & $* *$ & $* *$ & $* *$ & $* *$ & $* *$ & $* *$ & $* *$ & $* *$ & $* *$ & $* *$ \\
\hline $\mathbf{K}_{\mathbf{0}}$ & 16.696 & 16.75 & 3.87 & 3.95 & 43.13 & 43.19 & 269.71 & 268.07 & 83.33 & 82.52 \\
\hline$K_{50}$ & 18.676 & 18.97 & 4.25 & 4.29 & 45.18 & 45.71 & 289.07 & 292.71 & 86.76 & 86.69 \\
\hline F.test & $* *$ & $* *$ & $* *$ & $* *$ & $* *$ & $* *$ & $* *$ & $* *$ & $* *$ & $* *$ \\
\hline Sids 7 & 19.51 & 19.59 & 4.32 & 4.37 & 53.02 & 53.13 & 242.66 & 240 & 87.86 & 87.38 \\
\hline Giza 168 & 15.86 & 16.13 & 3.81 & 3.87 & 35.27 & 35.78 & 316.12 & 320.79 & 77.12 & 75.53 \\
\hline F.test & $* *$ & $* *$ & $* *$ & $* *$ & $* *$ & $* *$ & $* *$ & $* *$ & $* *$ & $* *$ \\
\hline Dx N & $* *$ & $* *$ & $* *$ & $* *$ & $* *$ & *** & *** & $* *$ & *** & $* *$ \\
\hline D X K & $* *$ & $* *$ & $* *$ & $* *$ & $* *$ & $* *$ & $* *$ & $* *$ & $* *$ & $* *$ \\
\hline $\mathbf{N} \times \mathbf{K}$ & $*$ & $* *$ & $* *$ & $* *$ & $* *$ & $* *$ & $* *$ & $* *$ & $* *$ & $* *$ \\
\hline D x V & $* *$ & $* *$ & $* *$ & $* *$ & $* *$ & $* *$ & $* *$ & $* *$ & $* *$ & $* *$ \\
\hline $\mathbf{N} \times \mathbf{V}$ & $* *$ & $* *$ & $*$ & $*$ & $* *$ & $* *$ & $* *$ & $* *$ & $* *$ & $* *$ \\
\hline K x V & $* *$ & $* *$ & $* *$ & $* *$ & $* *$ & $* *$ & $* *$ & $* *$ & $* *$ & $* *$ \\
\hline$D \times N \times K$ & $* *$ & $* *$ & $* *$ & $* *$ & $* *$ & $* *$ & $* *$ & $* *$ & $* *$ & $* *$ \\
\hline $\mathrm{D} \times \mathrm{N} \times \mathrm{V}$ & $* *$ & $* *$ & $* *$ & $* *$ & $* *$ & $* *$ & $* *$ & $* *$ & $* *$ & $* *$ \\
\hline D $x$ K x V & $* *$ & $* *$ & $* *$ & $* *$ & $* *$ & $* *$ & $\mathrm{~ns}$ & $* *$ & $* *$ & $\mathrm{Ns}$ \\
\hline $\mathbf{V} \times \mathbf{K} \times \mathbf{N}$ & $* *$ & $* *$ & $* *$ & $* *$ & $* *$ & $* *$ & $* *$ & $* *$ & ns & Ns \\
\hline $\mathrm{D} \times \mathrm{N} \times \mathrm{K} \times \mathrm{V}$ & $* *$ & $* *$ & $* *$ & $* *$ & $* *$ & $* *$ & $* *$ & $* *$ & ns & $* *$ \\
\hline
\end{tabular}


Table 9. Effect of $\mathrm{N}, \mathrm{K}$ and timing of $\mathrm{N}$ application on harvest index (HI)

\begin{tabular}{|c|c|c|c|c|}
\hline \multicolumn{2}{|c|}{ Treatment } & Giza 168 & Sids 7 & Relative variation (\%) \\
\hline N level & 75 & 41.12 & 38.63 & -6.06 \\
\hline (Kg/fed) & 120 & 37.14 & 42.26 & +13.78 \\
\hline \multicolumn{2}{|c|}{ Relative variation (\%) } & -9.67 & +9.39 & \\
\hline \multirow{2}{*}{$\mathrm{Kg} \mathrm{K} \mathrm{K}_{2} \mathrm{O} / \mathrm{Fed}$. } & $\mathbf{0}$ & 38.75 & 39.98 & +3.17 \\
\hline & $\mathbf{5 0}$ & 39.48 & 40.19 & +3.62 \\
\hline \multicolumn{2}{|c|}{ Relative variation (\%) } & +1.88 & +2.31 & \\
\hline \multirow{7}{*}{ 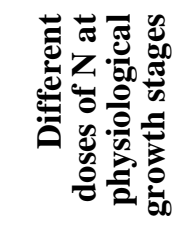 } & Cont. & 32.54 & 31.75 & -2.42 \\
\hline & $\mathbf{D}_{1}$ & 36.28 & 39.25 & +8.19 \\
\hline & $\mathbf{D}_{2}$ & 37.84 & 40.11 & +5.99 \\
\hline & $\mathbf{D}_{3}$ & 39.98 & 40.41 & +1.08 \\
\hline & $\mathbf{D}_{4}$ & 40.73 & 40.81 & +0.20 \\
\hline & $\mathbf{D}_{5}$ & 41.64 & 41.03 & -1.46 \\
\hline & $D_{6}$ & 39.75 & 40.45 & +3.37 \\
\hline
\end{tabular}

1000 grain weight:

Table 7 indicated that 1000 grain weight of the studied wheat varieties were highly significantly increased by increasing $\mathrm{N}$ level up to $120 \mathrm{Kg} \mathrm{N} /$ feddan. The highest mean values were 40.45 and $62.9 \mathrm{~g}$ for Giza 168 and Sids 7, respectively. This result was similarly observed by Muhammad (2001) and El Naggar (2003).

Data revealed that 1000 -grain weight of the studied wheat varieties was highly significantly increased in the presence of potassium and the highest mean value was recorded under Sids 7. This result was similarly observed by Amer (2009)

Data showed that time of $\mathrm{N}$ application have a high significant effect on 1000 grain weight of the studied wheat varieties. The highest mean values were recorded at $\mathrm{D}_{3}(15: 30: 15: 15),(24: 30: 33: 33)$ for both $\mathrm{N}_{75}$ and $\mathrm{N}_{120}$ (38.31, 40.45) with Giza 168, while Sids 7 recorded highest mean value at $\mathrm{N}_{75} \mathrm{D}_{4}(15: 40: 10: 10)$ and $\mathrm{N}_{120} \mathrm{D}_{3}$. The values were $\left(57.0\right.$ and $62.75 \mathrm{~g}$ ) for $\mathrm{N}_{75}, \mathrm{~N}_{120}$. Data indicated there were high significant effects on 1000 grain weight due to the interaction between treatments (Table 8). This result was similarly observed by El Kholy (2000).

\section{Effect of $N$, $K$ and timing of $N$ application on harvest index $(\mathrm{HI})$ :}

Table (9) indicated that HI decreased with increasing $\mathrm{N}$ level with Giza 168 while it increased with Sids 7 by about $9.39 \%$ by increasing $\mathrm{N}$ level from 75 to $120 \mathrm{Kg} /$ feddan. Data showed that time of $\mathrm{N}$ application have a high significant effect on $\mathrm{HI}$ and recorded high value under $\mathrm{D}_{5}, \mathrm{D}_{6}$ for Giza 168 and Sids 7 This result was similarly observed by Wagan et al. (2002). HI was also, significantly affected by $\mathrm{K}$ application. Similar results were reported by Singh and Pathak (2003).

\section{REFERENCES}

A.O.A.C. (1980) Association of official Agriculture chemists. (Official methods of analysis), $13^{\text {th }}$ Ed., Washington, D.C.
Abd Allah, A. A. S. K. Badr and A.M.Ali (1999). Response of some wheat cultivars to potassium fertilization . Monoufia Journal of Agric . Res. 24 (3): 869-880,June. 1999.

Abd El - Wahed A. E. M (1997). Effect of seeding rate and N Ferilization level on yield and yield atributes of some Egyptian bread. J. Agric. Res . Tanta univ., 23 (4): 359 368 Dec. 1997.

Amer, M..M..A (2009) Response of Wheat Yield to Fertilization by Nitrogen, Potassium and Biofertilizers in Salt Affected Soils Ph. D Thesis; Soils, Fac Agric. Kafr El - Sheikh univ., Egypt .

Cochran, W.G. and, G. M. Cox (1960). Experimental Designs, 2 ndded John welly , Newyork,: 293-316.

Cox-W.J; G.G-Bergstrom; WS-Ried; ME-Sorrels and D-Otis (1989). Fungicide and $\mathrm{N}$ effects on winter wheat under low foliar disease severity-crop-Science. 1989, 29:1, 164170; 29 ref.

Eisa, F. A. (1996). Summary agronomic studies on wheat, $\mathrm{Ph}$. D. Thesis; Agronomy, Mansoura Univ. Fac. Of Agric library 633.11

El - Banna A. Y. A. ( 2000 ). Effect of seeding rates and PK fertilizer levels on grain yield and yield attributes of wheat under newly cultivated sandy soil conditions - Zagazig J . of Agric Res. 27 (5): 1161 - 1178 , sep 2000.

El - Desokey, M. A.; A. Ghallab and E. A. Teama (2000). Efficient use and uptake of nitrogen for wheat yield in a clay soil in relation to split nitrogen application. Assiut- J. of-Agric. Sci. 2000, 31:5, 153-167; 23 ref.

El - Desouqi, S. A. (2000). Effect of some nitrogen sources on growth and yield of wheat plants. Conf. International Colloquium for the optimization of plant nutrients, yield quality, the environment. 10th . Cairo (EGY). Apr. 8-13, 2000.

El - Far, I.. A. and G. R. El-Nagar, (1995). Influence of time of nitrogen application on yield, yield components and quality of two wheat cultivars. Assiut J. of Agric. Sci. 26 : 2, 106-113, 1995.

El - Naggar; G. R. (2003). Yield and quality of some spring wheat genotypes subjected to different nitrogen fertilizer rates Assiut journal of Agric Sci . 34 : 32, 43-63,2003. 
El - Sherbieny A. E.; K. G. Soliman and R. M. Ali (1999). Increasing the efficiency of nitrogen fertilizers in newly reclaimed sandy soil. Zagazig J. Agric. Res. 26 : 38, 895906.

El Kholy M. A. (2000). Response of wheat growth and yield to plant Density and methods of nitrogen and potassium fertilizers application Egypt. J. Agron. Vol. 22, pp 1-18 .

Ewert, F. and B. Honermeier (1999). Spikelets inition of winter triticale and winter wheat in response to nitrogen fertilization. European J. Agron. 11, 107-113, 1999. (c.f. Mengel, K. and E. A. Kirkby (2001).

Faizy, S. E. D. A.; M. S. Shams; M. M. Rizk and A. M. Hamissa (1986a). Effect of the rate and time of urea application on yield and yield components of wheat plant. J. Agric. Res. Tanta Univ., 12(4):1205-1217.

Faizy, S. E. D. A.; T. M. El-Essawi; R. A. Ali and A. ElShamly (1986b). Spliting nitrogen and the effect of potassium fertilizer on grain yield of wheat, . J. Agric. Res. Tanta Univ., 12(4):1241-1247.

Forster, H. (1973). Effect of potassium and nitrogen supply to plants on yield components and yield formation of cereals. Landw. Forsch. 26:221-227. (c. f. Mengel, and E. A. Kirkby (2001).

Genaidy, S. A. and M.A Hegazy (2001). Evaluation for effects of potassium fertilization from sulfate and chloride sources on some main field crops Egypt. J. Agric . Res.,79(2),2001.

Gohar, A. T. (1954). The influence of Exchangeaple cations on physical properties of Egyptian soil M. Sc thesis, Fac. of Agric. Cairo univ.

Jackson , M.L. (1967). Soil chemical Analysis prentice Hall of India Ltd New Delhi.

Koreish. E. A., M. E. El-Fayoumy, H. M. Ramadan, and W. H. Mohamed (2004), Interaction Effect of organic and mineral fertilization on Falabean and wheat productivity in calcareous soils. Alex. J. Agric. Res. 49 (2) : 101 - 114, 2004.

Maqsood. M; A.Ali; A.Zubair; M. Saeed and S.Ahmad (2002). Effect of irrigation and nitrogen levels on grain yield and quality of wheat (Triticum aestivum). International J. of Agric. and Biology 2002, 4:1, 164-165; 8 ref.

Mengel; K and E. A. Kirkby (2001). Principles of plant Nutrition 5th Edition KLUWER ACADEMIC publishers Dorotrecht / Boston / London.
Muhammad ; N.A.(2001). Curve analysis for evaluation of the response of some wheat varieties to different nitrogen fertilization levels . Mansoura university Journal of Agric .Sci . 24 (4), 1559-1571,Apr 199.

Olsen, S.R.; C. V. Cale; F. S. Watenable and L. A. Dean (1954). Estimation of available phosphorus in soil by extraction with sodium bicarbonate U. S. Dept., Agric. Circ., 939.

Ooro-PA and H.G Mwangi (1999). The effects of nitrogen rate and application timing on the yield of bread wheat. Proceedings of the tenth Regional Wheat Workshop for Eastern, Central and Southern Africa, University of Stell enbosch, South Africa, 14-18 September, 1998. 1999, 335-337; 3 ref.

Page, A. L. (1982) . Methods of soil analysis. Part 2: Chemical and micribiological properties ( $2^{\text {nd }}$ ed.) Amer. Soc. Agron. In Soil Sci. Soc. Amr. Inc. Madison, Wisconsin, USA.

Piper, C. S. (1950). Soil and plant analysis. Inter science Publication. New York.

Rahman, M. A. ; M. A. Sufian; M. Saifuzzaman and J. Chikushi (2002). Nitrogen management in rice-wheat alternating cropping system and wheat genotype identification preferable to surface seeding condition. J.of-the - Faculty-of Agric. Kyushu-Univ. 2002, 46:2,295$301 ; 13$ ref.

Sabry, S. R. S. ; E. M. Taha and A. A. Khattab (1999). Response of long spike wheat (Triticum aectivum L.) genotypes to nitrogen fertilizer levels in soils of middle Egypt. Bulletin of faculty of Agric. Univ. of Cairo. 1999, 50:2, 169-188, 20 ref.

Schacherer ,A and H.G.Beringer (1984). Number and size distribution . of endo . sperm cells in developing cereals grains as an index for their sink capacity. Ber Deutsch. Botgas . 97,183-195,1984.

Sing.R.N and R.K.Pathak (2003) Effect of potassium and magnesium on yield, their uptake and quality characteristics of wheat (Triticum aestivum). Journal of the Indian society of soil science 2003, 2, 181-185; 21 ref.

Snell, F. D. and C. T. Snell (1967). Colorimetric methods of analysis. D. Van. Nostranad company Inc.: $551-552$.

Wagan M. R; F. C . Oad and K. S. Nenwani ( 2002 ). Wheat growth and yield contributing characters under various sources and schedules of nitrogen fertilizer Pakistan - J. of - Applied - sciences. 2002, 2:11, $1013-1015 ; 13$ ref .

Warraich, E. A.; Nazik-Ahmed; S. M. A. Basra and IrfanAfzal (2002). Effect of nitrogen on source-sink relationship in wheat. International- J. of-Agric.-and Biology. 2002, 4:2, 300-302; 34 ref. 


\section{الملخص العربي}

\section{تأثير إضافة التسميد الأزوتى والبوتاسى فى مراحل النمو الفسيولوجية على محصول صنفين من القمح النامية في شمال وسط الدلتا}

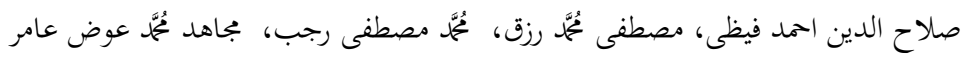

دفعات (24 :60 : 24 : 18: 18:)، مع إضافة 50 كجم (بو2 أ)، 22

$$
\text { كجم (فوأك). }
$$

تشير النتائج إلى زيادة معنوية في عدد الحبوب في السنبلة لصنفي

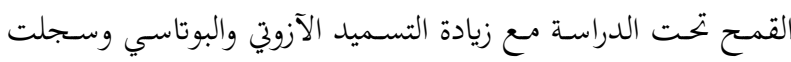

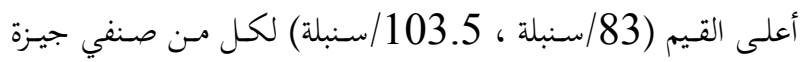

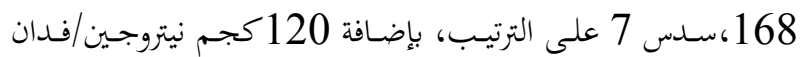

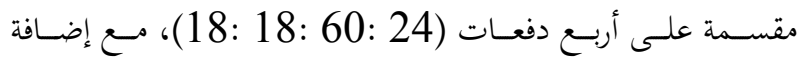
50 كجم (بو2 أ) في وجود التسميد الفوسفاتي 22 كجم (فوأك). تشير النتائج إلى زيادة معنوية في وزن الألف حبة لأصناف القمح

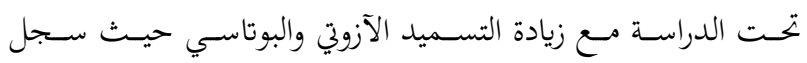

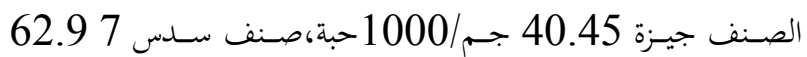

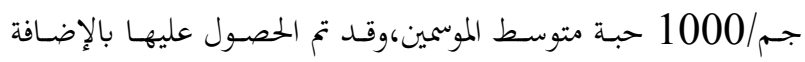

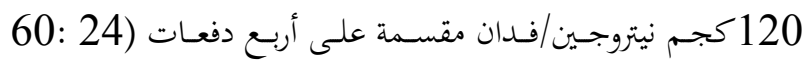

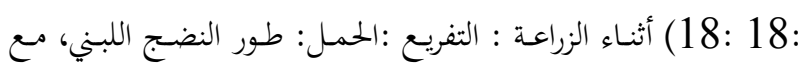

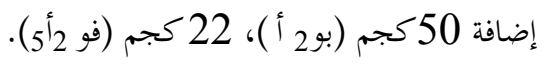

تبين مـن النتائج أنة يمكـن الحصول على أعلى وزن الألف حبـة

54جم بإضافة 36كجم أزوت/فدان بدون إضافة البوتاسيوم، بينما

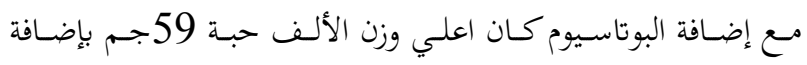

$$
\text { 29كجم أزوت/فدان. }
$$

سجل دليل الحصاد أعلى القيم (41.12\%) لصنف جيزة 168

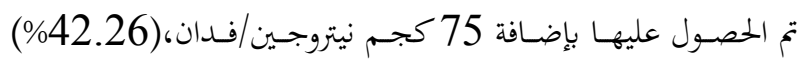

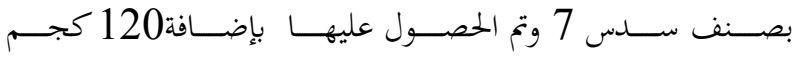

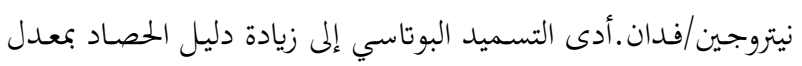

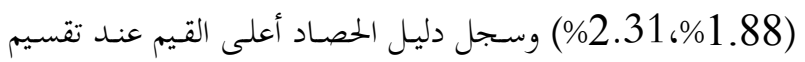
السـماد النيتروجيـني كالتـالي (24: 50: 23: 23 ) ) مـع الزراعـة:
تم إجراء تجرب حقلية بمزرعة كلية الزراعة بكفر الشيخ خلال موسمى2003/2002،2004/2003حيث كان الهدف دراسة تأثير إضافة الأزوت في مرحلة التفريع على محصول القمح وبعض مكوناته وكذلك التسميد البوتاسى،وقد لخصت النتائج المتحصل عليها فيما يلي: تشير النتائج إلى أن معدلات إضافة النيتروجين لها تأثير معنوي

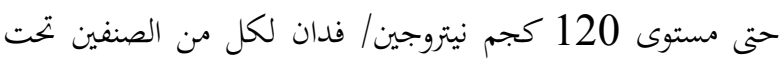
الدراسة وكانت أعلى قيمة للمحصول (متوسط الموسمين) سجل الصنف جيزة $168 \quad 21.91$ إردب/فدان، وسدس 7 (30.0أردب/فدان)تم الحصول عليها بالإضافة 120كجم نيتروجين للفدان مقسمة كالتالي(24: 60: 18:18) كجم نيتروجين للفدان

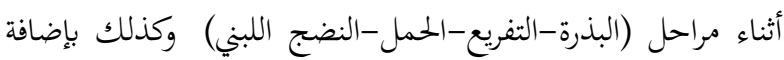

$$
50 \text { كجم (بو2 أ)، 22 كجم (فوأك ) مع الزراعة. }
$$

توضح النتائج أن معدلات إضافة النيتروجين لها تأثير معنوي على قش القمح حتى معدل 120كجم نيتروجين/فدان حيث سجل الصنف جيزة 1685.19 طن/فدان،سدس 67 67طن/فدان، متوسط الموسمين تم الحصول عليها بإضافة 120كجم نيتروجين/فدان (24)

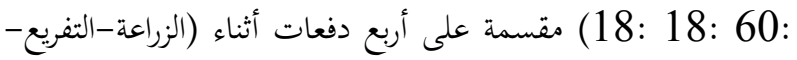

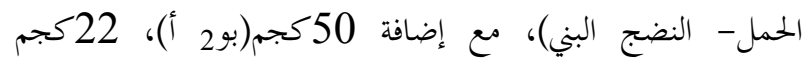

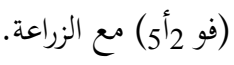
تبين النتائج إلى زيادة معنوية في عدد السنابل في المتر المربع مع الزيادة في معدل النيتروجين المضاف وكذلك التسميد البوتاسي وكانت أعلى قيمة في عدد السنابل المتر المربع متوسط الموسمين/ 435 سنبلة/22 لصنف جيزة 168، 300سنة 300بلة/ק2صنف سدس 7 تم الحصول عليها مع إضافة120كجم نيتروجين/فدان مقسمة على أربع 
120 كجم نيتروجين/فدان بمعلات: (18:18:60:24) خـالال

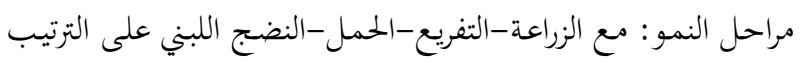

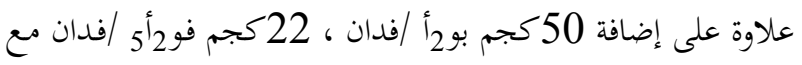

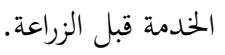

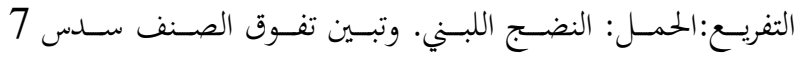
(40.45\%) على صنف جيزة 168(39.13\%) للحصول على أعلى محصول من الحبوب والقش لأصناف القمح

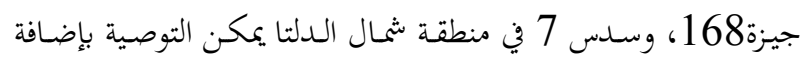

